dex for cattle. Genome Res. 11:626-630.

4.Takasuga, A., S. Hirotsune, R. Itoh, A. Jitohzono, H. Suzuki, H. Aso, and Y. Sugimoto. 2001. Establishment of a high throughput EST sequencing system using poly(A) tail-removed cDNA libraries and determination of 36000 bovine ESTs. Nucleic Acids Res. 29:e108.

5.Cicera, S., A.K. Wintero, and M. Fredholm. 2000. Why do we still find anonymous EST? Mamm. Genome 11:689-693.

6.Sambrook, J. and D.W. Russel. 2001. Molecular Cloning: A Laboratory Manual, 3rd ed. CSH Laboratory Press, Cold Spring Harbor, NY.

7.Endege, W.O., K.E. Steinmann, L.A. Boardman, S.N. Thibodeau, and $R$. Schlegel. 1999. Representative cDNA libraries and their utility in gene expression profiling. BioTechniques 26:542-550.

8.Bédard, J., S. Brûlé, C.A. Price, D.W. Silversides, and J.G. Lussier. 2003. Serine protease inhibitor-E2 (SERPINE2) is differentially expressed in granulosa cells of dominan follicle in cattle. Mol. Reprod. Dev. 64:152 165

9.Zhumabayeva, B., C. Chang, J. McKinley, L. Diatchenko, and P.D. Siebert. 2001. Generation of full-length cDNA libraries enriched for differentially expressed genes for functional genomics. BioTechniques 30:512-520.

10.Franz, O., I. Bruchlaus, and T. Roeder 1999. Verification of differential gene transcription using virtual blotting. Nucleic Acids Res. 27:e3.

11.Villalva, C., C. Touriol, P. Seurat, P. Trempat, G. Delsol, and P. Brousset. 2001. Increased yield of PCR products by addition of T4 gene 32 protein to the SMART ${ }^{\mathrm{TM}}$ PCR cDNA synthesis. BioTechniques 31:81-86.

12.Inoue, H., H. Nojima, and H. Okayama. 1990. High efficiency transformation of $E s-$ cherichia coli with plasmids. Gene 96:23-28.

13.Rabahi, F., S. Brûlé, J. Sirois, J.-F. Beckers, D.W. Silversides, and J.G. Lussier. 1999. High expresssion of bovine $\alpha$ glutathione Stransferase (GSTA1, GSTA2) subunits is mainly associated with steroidogenically active cells and regulated by gonadotropins in bovine ovarian follicles. Endocrinology 140:3507-3517.

14.Liang, P. 2002. A decade of differential display. BioTechniques 33:338-344

Received 24 January 2003; accepted 23 March 2003.

Address correspondence to Jacques G. Lussier, Faculté de médecine vétérinaire, Université de Montréal, P.O. Box 5000, StHyacinthe, QC, Canada, J2S 7C6. e-mail: jacques.lussier@umontreal.ca

\section{Dictyostelium discoideum transformation by oscillating electric field electroporation}

\author{
Laeticia Alibaud ${ }^{1}$, Pierre Cosson ${ }^{1}$, and Mohammed Benghezal ${ }^{2}$ \\ ${ }^{1}$ Université de Genève, ${ }^{2}$ Athelas SA, Geneva, Switzerland
}

BioTechniques 35:78-83 (July 2003)

Dictyostelium discoideum has been used as a genetically tractable model organism to study many biological phenomena. High-efficiency transformation is a prerequisite for successful genetic screens such as mutant complementation, identification of suppressor genes, or insertional mutagenesis. Although exponential decay electroporation is the standard transformation technique for $\mathrm{D}$. discoideum, its efficiency is relatively low and its reproducibility is weak. Here we optimized the oscillating electroporation technique for $\mathrm{D}$. discoideum transformation and compared it to the exponential decay electroporation. A 20-fold increase in the efficiency was reproducibly achieved. This alternative electroporation technique should facilitate future genetic approaches in $\mathrm{D}$. discoideum.

\section{INTRODUCTION}

The social amoeba Dictyostelium discoideum lives in the soil as a unicellular organism feeding on bacteria. Upon starvation, Dictyostelium amoebae initiate a development program that leads to the formation of a multicellular organism and fruiting bodies containing spores (1). With a haploid genome of $34 \mathrm{Mb}, D$. discoideum represents a powerful genetic system to investigate many biological processes such as cell motility and development (2), phagocytosis and endocytosis (3), and host- pathogen interaction (4-7). Therefore, the availability of an efficient transformation technique is desirable to perform genetic screens, such as cDNA suppressor isolation or insertional mutagenesis, or to generate mutants by homologous recombination. Exponential decay electroporation has been used to introduce DNA molecules into $D$. discoideum cells for more than a decade (8). A significant increase in the transformation efficiency would help researchers and bring $D$. discoideum genetics to its full potential. A recent study demonstrated that $D$. discoideum

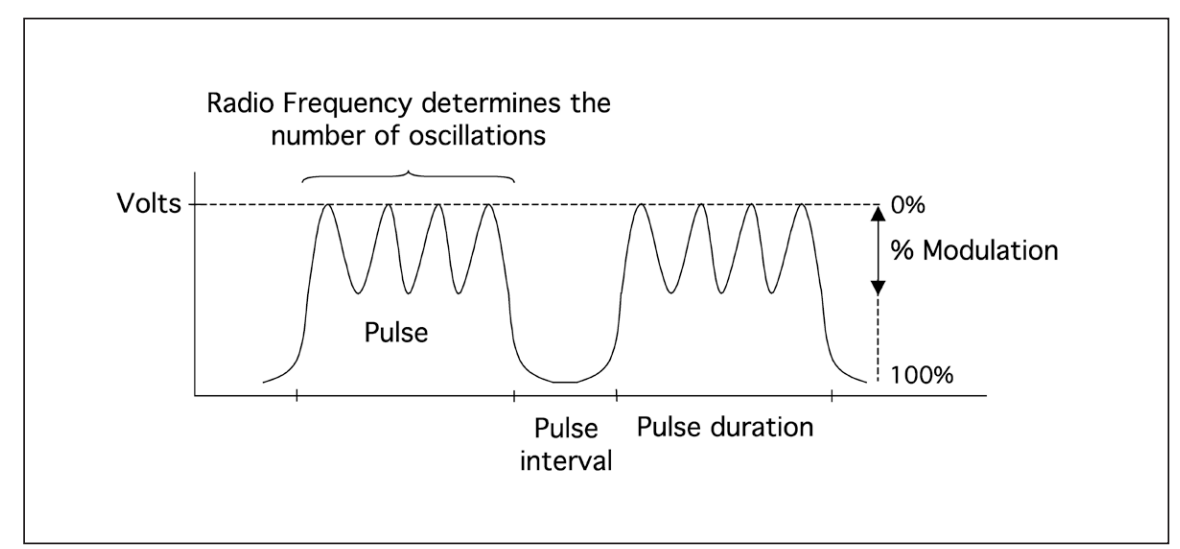

Figure 1. Scheme of the oscillating electric field electroporation. The electric field is applied as a succession of pulses. A rectangular pulse is obtained when modulation is set to $0 \%$, and an oscillating pulse when modulation is set to $100 \%$. Different parameters can be regulated: voltage, pulse duration, number of pulses, RF, pulse-interval duration, and percent of modulation. 
A

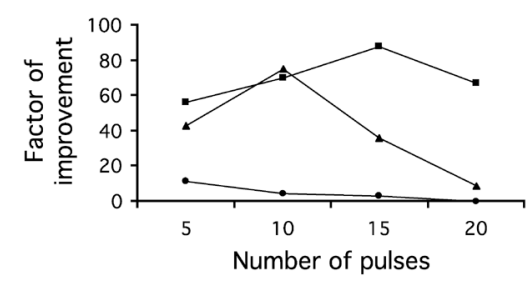

B

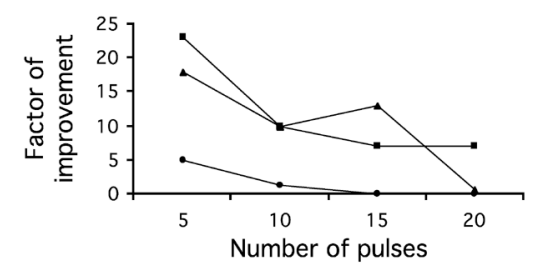

C

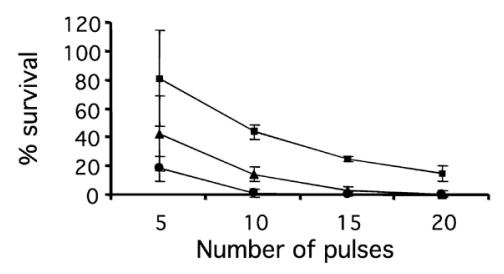

D

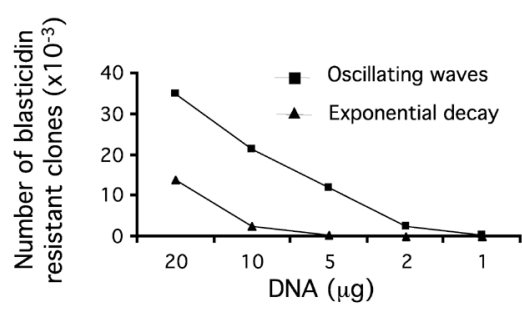

Figure 2. Optimization of the oscillating electric field electroporation. The following variables were kept constant: $400 \mathrm{~V}, 100 \%$ of modulation, RF of $50 \mathrm{~Hz}$, and pulse interval of $1 \mathrm{~s}$. (A and B) Transformation efficiencies compared to the exponential decay electroporation (two separate experiments). The factor of improvement was defined as the ratio of the number of blasticidin-resistant transformants of the oscillating electroporation technique and the number of transformants of the exponential decay electroporation procedures. The number of pulses was varied from 5 to 20 for each pulse duration (square, $2 \mathrm{~ms}$; triangle, $3 \mathrm{~ms}$; and circle, $4 \mathrm{~ms}$ ). Ten micrograms of DNA were used. (C) Cell viability (\% survival) after oscillating electroporation was determined by counting plaque formation on a $K$. pneumoniae lawn. The number of pulses was varied from 5 to 20 for each pulse duration (square, $2 \mathrm{~ms}$; triangle, $3 \mathrm{~ms}$; and circle, $4 \mathrm{~ms}$ ). Ten micrograms of DNA were used. Means and standard deviations from three independent experiments are represented. (D) Titration of DNA amounts in oscillating and exponential decay electroporation procedures. The pulse duration and number of pulses were set constant to $2 \mathrm{~ms}$ and 5 pulses, respectively. cell transformation could be improved by using a particle inflow gun (9). A new electroporation technique has been reported for mammalian cells (10-12). In this technique, the conventional direct current electric field is shifted by a radio frequency (RF) electric field. This results in a succession of oscillating pulses instead of a unique pulse of exponential decay (Figure 1). Many parameters can be modulated: voltage, pulse duration, number of pulses, RF, pulse-interval duration, and percent of modulation. This latter parameter allows the application of pulses from rectangular pulses like square wave $(0 \%$ modulation) to sinusoidal pulses $(100 \%$ modulation), with the maximum voltage at the selected voltage and the minimum voltage at $0 \mathrm{~V}$ (Figure 1).

Here we optimized the oscillating electric field electroporation technique for $D$. discoideum cell transformation and obtained a significant increase in the efficiency of transformation, compared to the exponential decay electroporation technique.

\section{MATERIALS AND METHODS}

\section{Strains and Culture Condi- tions}

The subclone DH1.10 (13) of the $D$. discoideum wild-type strain DH1 (14) was used in this study. Cells were grown at $21^{\circ} \mathrm{C}$ in HL5 medium containing 14.3 g/L peptone (Oxoid LTD, Basingstoke, Hampshire, UK), 7.15 g/L yeast extract (Brunschwig BD Difco, Basel, Switzerland), $18 \mathrm{~g} / \mathrm{L}$ maltose (Fluka, Buchs, Switzerland), $3.6 \mathrm{mM} \mathrm{Na}_{2} \mathrm{HPO}_{4}$, and $3.6 \mathrm{mM} \mathrm{KH}_{2} \mathrm{PO}_{4}, \mathrm{pH} 6.7$.

Klebsiella pneumoniae was used as a growth substrate when cells were plated on SM agar [10 $\mathrm{g} / \mathrm{L}$ peptone, $1 \mathrm{~g} / \mathrm{L}$ yeast extract, $16.2 \mathrm{mM} \mathrm{KH} \mathrm{KO}_{4}, 5.7 \mathrm{mM}$ $\mathrm{K}_{2} \mathrm{HPO}_{4}, 4 \mathrm{mM} \mathrm{MgSO}, 1 \%$ glucose, and $20 \mathrm{~g} / \mathrm{L}$ bactoagar (Brunschwig DB Difco)].

\section{Electroporation Procedure}

Cells were transformed with the integrative vector $\mathrm{pUCBs} \Delta \mathrm{Bam}$ encoding the blasticidin selective marker (15). DNA was purified with the Endofree ${ }^{\circledR}$ Plasmid Maxi Kit (Qiagen, Basel, Switzerland) and resuspended in endotoxinfree water. After 10 min of incubation on ice, the cells were washed once with sterile ice-cold electroporation buffer (10 mM NaPO 4, pH 6.1, $50 \mathrm{mM}$ sucrose) and resuspended to $5 \times 10^{7}$ cells $/ \mathrm{mL}$ in the same buffer. Nonlinearized DNA was added at the indicated concentrations, and $0.4 \mathrm{~mL}\left(2 \times 10^{7}\right.$ cells) of the suspension was transferred into the electroporation cuvette (Gene Pulser ${ }^{\circledR}$ Cuvette, 0.2-cm gap; Bio-Rad Laboratories, Hercules, CA, USA). Cells were electroporated at $0.8 \mathrm{kV}, 3$ $\mu \mathrm{F}$ using the Gene Pulser II system (Bio-Rad Laboratories) when a pulse of exponential decay was applied. These conditions have been previously optimized in our laboratory (unpublished results). Oscillating electric field electroporation was performed with the RF module (Bio-Rad Laboratories) connected to the Gene Pulser II system. The RF module delivers pulses of $400 \mathrm{~V}$ maximum. Immediately after the electroporation, the cells were processed as described below to evaluate the cell viability and the transformation efficiency.

Cell viability. One hundred of the 2 $\times 10^{7}$ electroporated cells were distributed together with $K$. pneumoniae onto SM agar plates. The percentage of survival was estimated by counting the number of $D$. discoideum plaques growing on a $K$. pneumoniae lawn. In the case of $100 \%$ viability, 100 clones should grow, and none should grow in the case of $0 \%$ viability.

Transformation efficiency. Onehundredth of the electroporated cells (2 $\times 10^{5}$ of $2 \times 10^{7}$ ) were distributed in a 9$\mathrm{cm}$ Petri dish containing $10 \mathrm{~mL}$ HL5 medium. Blasticidin $\mathrm{S}$ hydrochloride (10 $\mu \mathrm{g} / \mathrm{mL}$; ICN Biomedicals, Aurora, $\mathrm{OH}, \mathrm{USA}$ ) was added $24 \mathrm{~h}$ later. After 5 days incubation with no further manipulation of the Petri dish, visible colonies of transformants attached to the plastic were counted. Each colony corresponds 
to one independent clone. To obtain the absolute number of transformants per electroporation, the number of colonies has to be multiplied by 100 .

\section{RESULTS AND DISCUSSION}

The oscillating electric field electroporation parameters (Figure 1) were optimized for $D$. discoideum stable transformation with the integrative nonlinearized vector pUCBsr $\Delta \mathrm{Bam}$. The RF and the pulse-interval duration were always set to $50 \mathrm{~Hz}$ and $1 \mathrm{~s}$, respectively. The pulse duration and number of pulses were optimized in a first set of four independent experiments where the voltage and the modulation were kept constant at $400 \mathrm{~V}$ and $100 \%$, respectively. These fixed parameters had been determined as close to optimum in preliminary experiments. In parallel, the classical exponential decay electroporation was performed to compare both electroporation methods with the very same batch of cells and DNA suspension. Although some variability was observed between the four independent experiments, the oscillating electric field electroporation always gave a higher transformation efficiency compared to the exponential decay electroporation procedure (Figure 2). Overall, the latter technique gave rise to 100-1000 clones per electroporation, whereas the oscillating electric field technique gave rise to $7000-28,000$ clones. Figure 2, A and B, shows the oscillating electric field transformation efficiency relative to the exponential decay in two separate experiments. Two milliseconds of pulse duration appeared to be optimal, as the relative transformation efficiency ranged from 24 - to 90 -fold. Increasing the number of pulses from 5 to 20 did not give reproducible results. Indeed, a number of pulses higher than five sometimes gave very high efficiencies (Figure 2A) but also very poor efficiencies (Figure $2 \mathrm{~B}$ ). Altogether, in the four separate experiments, setting the pulse duration to 2 $\mathrm{ms}$ and the number of pulses to 5 resulted in a higher transformation efficiency that was reproducible. These conditions are now used in our laboratory, and at least 20-fold higher trans-

Table 1. Optimization of the Voltage and Modulation of the Oscillating Electric Field Electroporation

\begin{tabular}{|c|c|c|c|c|}
\hline \multirow[b]{2}{*}{$\%$ Modulation } & \multicolumn{4}{|c|}{ No. Blasticidin-Resistant Clones $\left(\times 10^{-3}\right)^{a}$} \\
\hline & $100 \mathrm{~V}$ & $200 \mathrm{~V}$ & $300 \mathrm{~V}$ & $400 \mathrm{~V}$ \\
\hline 100 & 0 & 0.9 & 21.5 & 28.6 \\
\hline 70 & 0 & 0.4 & 24.0 & 14.4 \\
\hline 30 & 0 & 8.6 & 4.7 & 0.3 \\
\hline 0 & 0 & 16.0 & 2.3 & 0.2 \\
\hline
\end{tabular}

formation rates are routinely obtained.

Figure 2C shows that cell survival decreased rapidly as pulse duration and the number of pulses increased. The highest cell viability was obtained for pulse duration of $2 \mathrm{~ms}$ and 5 pulses. Pulse duration other than $2 \mathrm{~ms}$ or number of pulses greater than 5 appeared to be detrimental to cell viability. Average cell viability in the classical exponential decay electroporation was $75 \%$. The cell viability correlated well with the reproducibility, as the more viable were the cells, the more reproducible were the efficiencies.

In a similar way, the voltage and the modulation were optimized by applying five pulses of $2 \mathrm{~ms}$. The highest efficiencies were obtained with the voltage and the modulation at $400 \mathrm{~V}$ (maximum capacity of the RF module) and $100 \%$, respectively (Table 1). These results confirm that the conditions set previously were optimal. Interestingly, the modulation and the voltage are tightly linked, as the best efficiencies are found on the diagonal (Table 1).

All the experiments described above were performed with $10 \mu \mathrm{g}$ DNA per electroporation. Titration of the DNA amount was then performed (Figure 2D). Transformation efficiencies were improved by increasing the amount of DNA in both electroporation procedures. However, oscillating electroporations required much lower amount of DNA to reach exponential decay efficiencies. This should be particularly appreciated when the DNA amount to be transformed is rate limiting. Indeed, DNA amounts are often a limiting factor (e.g., in the case of cDNA libraries).

Altogether, our results demonstrate that oscillating electric field electropo- ration is a more efficient technique than the classical exponential decay electroporation to transform $D$. discoideum cells. By optimizing the electroporation parameters (voltage, $400 \mathrm{~V}$; number of pulses, 5; pulse duration, $2 \mathrm{~ms}$; RF, 50 $\mathrm{Hz}$; pulse interval, $1 \mathrm{~s}$; modulation, $100 \%$ ), we were able to routinely obtain at least a 20 -fold higher transformation efficiency.

This new electroporation procedure for $D$. discoideum cell transformation should allow researchers to perform larger and more efficient genetic screens. Additionally, the many parameters of the oscillating electroporation technique are a great advantage for transformation optimization of different wild-type or mutant strains exhibiting sensitivity or resistance to electroporation.

\section{ACKNOWLEDGMENTS}

This work was supported by a START fellowship of the Fonds National Suisse de la Recherche Scientifique (FNRS) and a grant from the Fondation Gabriella Giorgio-Cavaglieri (both to P.C.).

\section{REFERENCES}

1.Kessin, R.H. 2001. Dictyostelium: Evolution, Cell Biology, and the Development of Cellularity. Cambridge University Press, Cambridge, UK.

2.Reymond, C.D., P. Schaap, M. Veron, and J.G. Williams. 1995. Dual role of cAMP during Dictyostelium development. Experientia 51:1166-1174.

3.Rupper, A. and J. Cardelli. 2001. Regulation of phagocytosis and endo-phagosomal trafficking pathways in Dictyostelium discoideum. Biochim. Biophys. Acta 1525:205216.

4.Cosson, P., L. Zulianello, O. Join-Lambert, 
F. Faurisson, L. Gebbie, M. Benghezal, C. Van Delden, L.K. Curty, and T. Kohler 2002. Pseudomonas aeruginosa virulence analyzed in a Dictyostelium discoideum host system. J. Bacteriol. 184:3027-3033.

5.Pukatzki, S., R.H. Kessin, and J.J. Mekalanos. 2002. The human pathogen Pseudomonas aeruginosa utilizes conserved virulence pathways to infect the social amoeba Dictyostelium discoideum. Proc. Natl. Acad. Sci. USA 99:3159-3164.

6.Solomon, J.M. and R.R. Isberg. 2000 Growth of Legionella pneumophila in Dictyostelium discoideum: a novel system for genetic analysis of host-pathogen interactions. Trends Microbiol. 8:478-480.

7.Solomon, J.M., A. Rupper, J.A. Cardelli, and R.R. Isberg. 2000. Intracellular growth of Legionella pneumophila in Dictyostelium discoideum, a system for genetic analysis of host-pathogen interactions. Infect. Immun. 68:2939-2947.

8.Knecht, D. and K.M. Pang. 1995. Electroporation of Dictyostelium discoideum. Methods Mol. Biol. 47:321-330

9.Wetterauer, B., K. Salger, P. Demel, and H. Koop. 2000. Efficient transformation of Dictyostelium discoideum with a particle inflow gun. Biochim. Biophys. Acta. 1499:139-143.

10.Chang, D.C. 1989. Cell poration and cell fu- sion using an oscillating electric field. Biophys. J. 56:641-652.

11.Chang, D.C., P.Q. Gao, and B.L. Maxwell. 1991. High efficiency gene transfection by electroporation using a radio-frequency electric field. Biochim. Biophys. Acta. 1092:153160.

12.Zheng, Q.A. and D.C. Chang. 1991. Highefficiency gene transfection by in situ electroporation of cultured cells. Biochim. Biophys. Acta 1088:104-110.

13.Cornillon, S., E. Pech, M. Benghezal, K. Ravanel, E. Gaynor, F. Letourneur, F. Bruckert, and P. Cosson. 2000. Phg1p is a nine-transmembrane protein superfamily member involved in Dictyostelium adhesion and phagocytosis. J. Biol. Chem. 275:3428734292

14.Caterina, M.J., J.L.S. Milne, and P.N. Devreotes. 1994. Mutation of the third intracellular loop of the cAMP receptor, $c A R 1$, of Dictyostelium yields mutants impaired in multiple signaling pathways. J. Biol. Chem. 269:1523-1532.

15.Adachi, H., T. Hasebe, K. Yoshinaga, T. Otha, and K. Sutho. 1994. Isolation of Dictyostelium discoideum cytokinesis mutants by restriction enzyme-mediated integration of the blasticidin S resistance marker. Biochem. Biophys. Res. Commun. 205:1808-1814.
Received 14 February 2003; accepted 25 March 2003.

Address correspondence to Mohammed Benghezal, Athelas SA, Rue Lombard, 15, Genève, CH-1205, Switzerland. e-mail: benghezal@athelas.com 